\title{
EFEITOS GENÉTICOS E DE AMBIENTE EM UM REBANHO DO ECÓTIPO MANTIQUEIRA. II. CARACTERÍSTICAS PRODUTIVAS ${ }^{1}$
}

\author{
Genetics and environment effects in mantiqueira type breed. II. Productive traits
}

\author{
Marcos Vinicius Gualberto Barbosa Silva², William José Ferreira ${ }^{3}$, Jaime Araujo Cobuci, \\ Paulo Rogério Palma de Oliveira ${ }^{5}$,Marco Antônio Machado ${ }^{2}$, Cezar Parreira Ferreira ${ }^{6}$
}

\begin{abstract}
RESUMO
Por meio de análises uni e bicaracterísticas entre a produção total de leite (PL), produção de gordura (PROG), porcentagem de gordura (PERG), duração da lactação (DL) e período seco (PSECO), objetivou-se, com este trabalho, estimar parâmetros genéticos utilizando-se registros provenientes das cinco primeiras lactações de 1.406 vacas do ecótipo Mantiqueira, filhas de 113 reprodutores, com partos entre os anos de 1977 e 1997, pertencentes ao programa de melhoramento do Pólo Regional do Vale do Paraíba da Agência Paulista de Tecnologia dos Agronegócios da Secretaria de Agricultura e Abastecimento do Estado de São Paulo (APTA/SAA-SP), no município de Pindamonhangaba. Os modelos usados para obtenção das médias dos quadrados mínimos incluíram os efeitos fixos de ano e estação de parto e idade ao parto, além dos efeitos aleatórios de reprodutor e do erro. Para a obtenção dos parâmetros genéticos pela metodologia REML foram utilizados os modelos que ajustavam os efeitos fixos de ano-estação e idade da vaca ao parto, além dos efeitos aleatórios de animal e do erro. As médias ajustadas \pm erros-padrão foram: $1863,31 \pm 1047,19 \mathrm{~kg}$ para a PL; 76,59 $\pm 31,92 \mathrm{~kg}$ para a PROG; 3,43 \pm 0,64 \% para PERG; 231,72 \pm 91,30 dias para DL; e 256,40 $\pm 180,29$ dias para o PSECO. As herdabilidades foram: 0,25; 0,09; 0,00; 0,16 e 0,06, respectivamente, para PL, PROG, PERG, DL e PSECO. As correlações genéticas entre PL e PROG ou PERG foram, respectivamente, de 0,36 e -0,02. De acordo com os resultados obtidos, pode-se inferir que há variação genética aditiva entre vacas para produção de leite.
\end{abstract}

Termos para indexação: Duração da lactação, ecótipo Mantiqueira, porcentagem de gordura, período seco, produção de gordura, produção de leite.

\begin{abstract}
Mantiqueira ecotype breed cows were analyzed to estimate genetic parameters for productive traits using single and multiple traits analysis of total milk production (PL), fat production (PROG), fat percentage (PERG), lactation length (DL) and days dry (PSECO). Records of the first five lactations of 1,406 cows from 113 sires with calving between 1977 and 1997 from the Paraiba Valley Regional Center of Research from São Paulo State (APTA/SAA-SP) breeding program were used. Models to obtain least square means included fixed effects for year and season of calving and age of calving, in addition to random effect of sire and error. The models to obtain the genetic parameters by REML methodology included fixed effects for year-season and age, in addition to random effects of animal and error. Least square means \pm standard error were 1863,31 $\pm 1047,19 \mathrm{~kg}(\mathrm{PL}) ; 76,59 \pm 31,92 \mathrm{~kg}(\mathrm{PROG}) ; 3,43 \pm$ $0,64 \%$ (PERG); 231,72 \pm 91,30 days (DL); and 256,40 $\pm 180,29$ days (PSECO). Estimated values of heritabilities were: 0.25 (PL); 0.09 (PROG); 0.00 (PERG); 0.16 (DL) and 0.06 (PSECO). Genetic correlations of PL with PROG and PERG were 0.36 and -0.02 , respectively. These results suggest an additive genetic variation among cows to milk yield.
\end{abstract}

Index terms: Days dry, fat percentage, fat yield, lactation length, Mantiqueira ecotype, milk yield.

(Recebido para publicação em 30 de abril de 2004 e aprovado 1 de setembro de 2005)

\section{INTRODUÇÃO}

A criação de raças especializadas para a produção de leite, como a Holandesa, pode ser uma das soluções para o incremento da produção de leite em países tropicais. Para tanto, programas racionais de manejo, alimentação, sanidade e melhoramento genético necessitam ser estabelecidos. Contudo, pode-se questionar que os desempenhos reprodutivos e produtivos das raças européias especializadas para a produção de leite em países tropicais sejam limitados pelo ambiente e manejo indesejáveis, apesar de, atualmente, haver consenso de que parte desta limitação estaria associada ao baixo nível tecnológico predominante (SILVA et al., 2001b).

Dessa forma, uma decisão importante é a definição dos sistemas de produção para as regiões tropicais e, portanto, a escolha do tipo de animal, dada a existência da interação genótipo-ambiente.

1 Instituto de Zootecnia - Parte integrante do projeto IZ - 14-001/52.

${ }^{2}$ Pesquisador da Embrapa Gado de Leite - Juiz de Fora, MG - marcos@cnpgl.embrapa.br

${ }^{3}$ Professor do Departamento de Zootecnia/DZO da Universidade Estadual de Santa Cruz - BA

${ }^{4}$ Professor da UFRGS - Departamento de Zootecnia - Av. Bento Gonçalves, 7712 - São José - 91.540-000 - Cx. P. 15.100 - Porto Alegre, RS

${ }^{5}$ Pesquisador Científico - Agência Paulista de Tecnologia dos Agronegócios/APTA - Secretaria da Agricultura e Abastecimento/SAA - Pindamonhangaba, SP.

${ }^{6}$ Aluno do Curso de Medicina Veterinária da UNIPAC - Bolsista de Iniciação Científica da FAPEMIG. 
Segundo Dickerson (1969), a variação genética entre as raças pode ser utilizada de três maneiras: i) seleção da melhor raça; ii) formação de nova raça; e iii) utilização permanente de cruzamentos. De acordo com esta classificação, o ecótipo Mantiqueira pode ser usado como uma nova raça pura ou em esquemas de cruzamento, em sistemas de produção de leite.

Como uma raça sintética, o ecótipo Mantiqueira apresenta como principal vantagem a facilidade prática de utilização de reprodutores, em comparação com outros esquemas de cruzamentos. Todavia, para poder utilizá-los, o criador deverá ter disponíveis reprodutores de bom valor genético, cuja determinação depende de um programa de avaliação genética baseado em teste de progênie.

Por outro lado, a tendência atual de utilização de raças naturalizadas em sistemas de produção é via cruzamentos com raças especializadas. Um bom exemplo desta tendência é, sem dúvida, a formação de compostos, já bastante difundida na pecuária de corte.

Os cruzamentos, segundo Teodoro \& Lemos (1992), são utilizados com a finalidade de reunir em um só animal as características desejáveis de duas ou mais raças, assim como explorar a heterose que é observada na maioria das características de importância econômica para os bovinos leiteiros nos trópicos. Ao se considerar as vantagens econômicas da utilização de cruzamentos, deve-se ter em mente que o mérito total é função de várias características, em que a heterose global é o acúmulo da heterose de cada característica (MADALENA, 1983). Também, sua expressão é dependente da distância genética entre as raças e das condições de ambiente a que estão sujeitos os animais.

Isto posto, objetivou-se, com este trabalho, estimar parâmetros genéticos de características produtivas do ecótipo Mantiqueira por meio de registros da produção total de leite (PL), produção de gordura (PROG), porcentagem de gordura (PERG), duração da lactação (DL) e período seco (PSECO).

\section{MATERIAL E MÉTODOS}

Os registros utilizados neste estudo foram provenientes das cinco primeiras lactações de 1.406 vacas do ecótipo Mantiqueira, filhas de 113 reprodutores, com partos entre os anos de 1977 e 1997. As informações referentes às características produção total de leite (PL), produção de gordura (PROG), porcentagem de gordura (PERG), duração da lactação (DL) e período seco (PSECO) foram obtidas do Arquivo de Escrituração Zootécnica do Pólo Regional do Vale do Paraíba da Agência Paulista de Tecnologia dos Agronegócios da Secretaria de Agricultura e Abastecimento do Estado de São Paulo (APTA/SAASP), no município de Pindamonhangaba.

O núcleo básico deste rebanho foi formado por meio da aquisição de matrizes e touros de quatro rebanhos de Minas Gerais e de um rebanho de São Paulo e, desde então, o rebanho foi fechado não sendo mais permitida a introdução de material genético de qualquer origem. Da formação do rebanho até o ano de 1974, simplesmente procurou-se ampliar o número de animais, não existindo um programa de acasalamento entre animais não aparentados. Em 1974, elaborou-se um plano, utilizandose quatro touros de origens distintas ("linhagens") e tendo como critério a não existência de avós em comum, o que foi feito até 1986, quando foram abertas mais seis "linhagens". Desde 1986, são selecionados, anualmente, dez reprodutores (um de cada "linhagem"), com base na primeira lactação de suas mães, desenvolvimento ponderal e tipo. Quanto às fêmeas, todas as novilhas eram incorporadas ao rebanho, desde que não possuíssem problemas reprodutivos ou defeitos anatômicos. Ressaltese que o direcionamento dos acasalamentos sempre foi feito mediante inspeção visual dos livros de escrituração zootécnica. Para maiores detalhes sobre o manejo adotado ver Silva et al. (2001a).

Para gerar os arquivos de análise foram descartadas observações com ordem de parto superior a cinco, duração da lactação inferior a 30 dias, bem como aquelas com produção de leite igual a zero, secagem anormal ou lactação em aberto. Também foram eliminados os registros com intervalo de partos inferior a 300 ou superior a 930 dias, ausência do ano de nascimento e pai desconhecido. Este último critério (pai desconhecido) só foi mantido nas análises realizadas para a obtenção das médias dos quadrados mínimos. Para melhor consistência dos dados, foi requerido que cada touro fosse pai, de pelo menos, quatro vacas. Ainda, cada nível de efeito fixo deveria conter, no mínimo, cinco informações.

Para obtenção das médias dos quadrados mínimos, os dados foram analisados utilizando-se o procedimento GLM (SAS INSTITUTE, 1985), cujos modelos estatísticos incluíram os efeitos fixos de ano e estação (estação seca abril a setembro e estação chuvosa - outubro a março) e idade da vaca ao parto, este último desdobrado em seus componentes linear e quadrático, além dos efeitos aleatórios de reprodutor e do erro. Os efeitos das interações ano*estação não foram significativos, sendo retirados das análises finais para todas as características estudadas.

Para a estimação dos parâmetros genéticos foram realizadas análises uni e bicaracterísticas envolvendo as

Ciênc. agrotec., Lavras, v. 30, n. 3, p. 529-537, maio/jun., 2006 
características produtivas. Utilizou-se o sistema MTDFREML (BOLDMAN et al., 1995), com modelos (modelo animal) que incluíram os efeitos fixos de anoestação de parto (águas - outubro a março, e seca - abril a setembro); como covariáveis, duração da lactação e idade da vaca no parto, em meses, com termos linear e quadrático, além dos efeitos aleatórios de animal, permanente de ambiente e erro. Matricialmente, os modelos para as análises bicaracterísticas podem ser representados por:

$$
\begin{gathered}
y_{1}=X_{1} \beta_{1}+Z_{1} u_{1}+P_{1} p_{1}+e_{1} \\
y_{2}=X_{2} \beta_{2}+Z_{2} u_{2}+P_{2} p_{2}+e_{2}
\end{gathered}
$$

tornando-se:

$$
\left[\begin{array}{l}
y_{1} \\
y_{2}
\end{array}\right]=\left[\begin{array}{cc}
X_{1} & 0 \\
0 & X_{2}
\end{array}\right]\left[\begin{array}{l}
\beta_{1} \\
\beta_{2}
\end{array}\right]+\left[\begin{array}{cc}
Z_{1} & 0 \\
0 & Z_{2}
\end{array}\right]\left[\begin{array}{l}
u_{1} \\
u_{2}
\end{array}\right]+\left[\begin{array}{cc}
P_{1} & 0 \\
0 & P_{2}
\end{array}\right]\left[\begin{array}{l}
p_{1} \\
p_{2}
\end{array}\right]+\left[\begin{array}{l}
e_{1} \\
e_{2}
\end{array}\right]
$$

em que:

$y_{1}$ e $y_{2}=$ vetores de $\mathrm{n} \times 1$ de observações para as características 1 e 2 , respectivamente;

$\beta_{1}$ e $\beta_{2}=$ vetores $\mathrm{p} \times 1$ de efeitos fixos, os quais incluíram os efeitos de ano-estação de parto e, como covariáveis, duração da lactação e idade da vaca no parto, em meses, com termos linear e quadrático, para as características 1 e 2, respectivamente;

$X_{1}$ e $X_{2}=$ matrizes de incidência n'p dos efeitos fixos para as características 1 e 2, respectivamente;

$u_{1}$ e $u_{2}=$ vetores $\mathrm{q} \times 1$ dos efeitos aleatórios de valor genético aditivo direto do animal, para as características 1 e 2 , respectivamente;

$Z_{1}$ e $Z_{2}=$ matrizes de incidência n'q dos efeitos aleatórios, para as características 1 e 2, respectivamente; $\mathrm{P}_{1}$ e $\mathrm{P}_{2}=$ matrizes de incidência dos efeitos de ambiente permanente, para as características 1 e 2 , respectivamente; $\mathrm{p}_{1}$ e $\mathrm{p}_{2}=$ vetores de efeitos de ambiente permanente do animal, para as características 1 e 2, respectivamente;

$e_{1}$ e $e_{2}=$ vetores $\mathrm{r} \times 1$ dos erros aleatórios, para as características 1 e 2, respectivamente;

As pressuposições adotadas foram:

$$
\begin{gathered}
E\left[\begin{array}{l}
y_{1} \\
y_{2}
\end{array}\right]=\left[\begin{array}{cc}
X_{1} & 0 \\
0 & X_{2}
\end{array}\right]\left[\begin{array}{l}
\hat{\beta}_{1} \\
\hat{\beta}_{2}
\end{array}\right] \\
\operatorname{Var}\left[\begin{array}{l}
y_{1} \\
y_{2}
\end{array}\right]=V \Rightarrow y \sim N(X \beta, V) \\
\operatorname{Var}\left[\begin{array}{l}
u_{1} \\
u_{2}
\end{array}\right]=G=\left[\begin{array}{ll}
A \sigma_{a 1}^{2} & A \sigma_{a 12} \\
A \sigma_{a 21} & A \sigma_{a 2}^{2}
\end{array}\right]
\end{gathered}
$$

$$
\begin{gathered}
\operatorname{Var}\left[\begin{array}{l}
p_{1} \\
p_{2}
\end{array}\right]=P=\left[\begin{array}{ll}
I \sigma_{p 1}^{2} & I \sigma_{p 12} \\
I \sigma_{p 21} & I \sigma_{p 2}^{2}
\end{array}\right] \\
\operatorname{Var}\left[\begin{array}{l}
e_{1} \\
e_{2}
\end{array}\right]=R=\left[\begin{array}{ll}
\sigma^{2}{ }_{e 1} I n & \sigma_{e 12} I n \\
\sigma_{e 21} I n & \sigma^{2}{ }_{e 2} I n
\end{array}\right]
\end{gathered}
$$

\section{RESULTADOS E DISCUSSÃO}

\section{Produção de leite}

A produção de leite é considerada, sob o ponto de vista do produtor, a mais importante característica para a avaliação produtiva de rebanhos leiteiros. De modo geral, a literatura é escassa no que se refere às pesquisas relacionadas com a produção de leite e seus componentes, em raças nativas (ou naturalizadas).

A média \pm desvio padrão (Coeficiente de variação CV) da produção total de leite (PL) de vacas do ecótipo Mantiqueira foi $1863,31 \pm 1047,19 \mathrm{~kg}$ (56,32\%). De modo geral, o resultado encontrado foi superior aos observados, no Brasil, por Jordão (1956) e Pereira (1993), na raça Caracu, e por Magofke et al. (1966), na raça Criollo Lechero de America Central, e Alba \& Kennedy (1995), na raça Criollo Lechero de Mexico, respectivamente, na Costa Rica e no México. Entretanto, foi inferior ao relatado por Magofke \& Bodisco (1966), na raça venezuelana Criollo Lechero Limonero.

Na TABELA 1 constam as médias de produção de leite segundo o ano de parto, na qual se verifica a grande oscilação dessas médias com o passar dos anos, em razão das variações nas condições de manejo e ambiente, porém com tendência de aumento na produção de leite. 
As médias ajustadas da produção de leite de acordo com a estação de parição encontram-se na TABELA 2, em que se verifica que as médias de PL de vacas que pariram na época das secas foram inferiores àquelas que pariram na época das chuvas, o que pode ter ocorrido em função da suplementação volumosa destinada aos animais na época seca do ano.

\section{Duração da Lactação}

Segundo Quirino et al. (1998), a baixa produtividade dos rebanhos leiteiros das raças nativas em regiões tropicais é, em parte, decorrente da baixa produção de leite e de durações de lactações mais curtas. As raças especializadas, normalmente, apresentam duração de lactação de 10 meses ou mais, enquanto nas raças naturalizadas, as lactações são mais curtas (QUIRINO, 1994).

O estudo da duração da lactação (DL) assume importância considerável por ser uma variável que influencia diretamente a produção total de leite do rebanho e, portanto, na lucratividade do sistema de produção. Do ponto de vista do melhorista, um problema no estudo de parâmetros genéticos e avaliação de animais de raças

TABELA 1 - Médias \pm erros-padrão (EP) da produção total de leite considerando as cinco primeiras lactações (PL), segundo o ano de parto.

\begin{tabular}{ccc}
\hline Ano de Parto & Número de Observações & PL $\pm \mathbf{E P}(\mathbf{k g})$ \\
\hline 77 & 121 & $1118,78 \pm 116,03$ \\
78 & 142 & $1728,78 \pm 107,06$ \\
79 & 80 & $1807,34 \pm 142,66$ \\
80 & 143 & $1571,22 \pm 107,09$ \\
81 & 159 & $1605,39 \pm 101,12$ \\
82 & 111 & $1703,08 \pm 121,02$ \\
83 & 122 & $1623,85 \pm 115,43$ \\
84 & 113 & $1788,76 \pm 119,95$ \\
85 & 163 & $1563,21 \pm 99,87$ \\
86 & 107 & $1468,44 \pm 123,26$ \\
87 & 95 & $1986,90 \pm 130,82$ \\
88 & 119 & $2096,30 \pm 117,19$ \\
89 & 92 & $2511,05 \pm 132,96$ \\
90 & 126 & $2535,93 \pm 113,64$ \\
91 & 175 & $2332,56 \pm 96,42$ \\
92 & 237 & $2579,94 \pm 82,82$ \\
93 & 211 & $2655,73 \pm 87,83$ \\
94 & 203 & $2335,07 \pm 89,49$ \\
95 & 282 & $2175,45 \pm 76,04$ \\
96 & 213 & $2130,84 \pm 87,43$ \\
97 & 130 & $2373,75 \pm 111,82$ \\
\hline
\end{tabular}

TABELA 2 - Médias \pm erros-padrão da produção total de leite na primeira lactação (PL1) e da produção total de leite considerando as cinco primeiras lactações (PL5) de vacas do ecótipo Mantiqueira, segundo a estação de parto.

\begin{tabular}{ccc}
\hline Estação de Parto & Número de Observações & PL \pm EP $(\mathbf{k g})$ \\
\hline Seca & 1636 & $1926,53 \pm 33,79$ \\
Chuvosa & 1511 & $2043,30 \pm 35,25$
\end{tabular}

Ciênc. agrotec., Lavras, v. 30, n. 3, p. 529-537, maio/jun., 2006 
nativas ou zebuínas é a decisão de eliminar ou não os registros de lactações curtas na preparação dos dados para a análise. Se a variação existente na duração da lactação não é de origem genética, torna-se interessante a adoção da padronização do período de produção, com eliminação de lactações curtas, geralmente consideradas anormais (MELLO, 1994). Todavia, as raças naturalizadas, nos trópicos, apresentam comumente lactações de duração inferior aos 305 dias, aceitas como meta para a obtenção de intervalos de parto de 12 meses. Como, durante as análises, foi observado que menos de $3 \%$ dos dados apresentavam períodos de lactação superiores a 305 dias, as lactações curtas não foram eliminadas (maiores que $30 \mathrm{e}$ menores que 120 dias), sendo consideradas como lactações anormais apenas aquelas inferiores a 30 dias.

A média \pm desvio padrão $(\mathrm{CV})$ da duração da lactação, no rebanho Mantiqueira, entre os anos de 1977 e 1997, foi de 231,72 \pm 91,30 dias (39,37\%). Considerando-se outros países de regiões tropicais, a duração média obtida neste estudo é semelhante às normalmente encontradas por outros autores, em raças crioulas latino-americanas, como as relatadas por Abreu (1988) e Magofke \& Bodisco (1966), na Venezuela, Bodisco \& Abreu (1981), na Colômbia, e por Pereira (1993) e Quirino (1994), no Brasil.

Todavia, deve ser considerado que, apesar de semelhante às outras raças, a duração da lactação média do ecótipo Mantiqueira é baixa, o que provavelmente se refletirá na lucratividade do sistema de produção. Assim, por apresentar baixa herdabilidade e considerável correlação genética com a produção de leite, a seleção indireta para DL, por meio da seleção direta para produção de leite, pode ser mais eficiente.

Em relação à duração da lactação de acordo com o ano de parição, houve grande oscilação das médias ao longo dos anos, não apresentando tendência de aumento ou diminuição, podendo-se inferir que a variação no manejo, principalmente o nutricional, deve ter atuado de forma marcante sobre essa característica.

Na Tabela 3, apresentam-se as médias de duração da lactação, segundo a estação de parto. Observa-se que as fêmeas paridas na estação chuvosa tenderam a apresentar lactações mais extensas. Esse resultado pode ter sido devido às condições favoráveis de crescimento das forragens na época das chuvas, resultando em lactações mais longas pelas boas condições corporais proporcionadas às fêmeas.

\section{Produção e Porcentagem de Gordura}

Apesar de sua importância para a avaliação genética, são escassos os trabalhos que tratam sobre a produção (PROG) e a porcentagem de gordura (PERG) de raças naturalizadas, no Trópico Americano. A maioria dos trabalhos encontrados foi realizada utilizando-se esquemas de cruzamento entre raças naturalizadas e especializadas, os quais mostraram que, de modo geral, a produção de gordura diminui com o aumento da proporção de genes da raça Holandesa e Pardo-Suíça, o que não ocorre nos cruzamentos com Jersey ou Guernsey (CUNNINGHAM \& SYRSTAD, 1987; PONCE \& BELL, 1981).

Quanto à seleção para essas características, Madalena (2000b) afirmou que os preços pagos ao produtor pela gordura e pela proteína são menores no Brasil do que nos principais países produtores de leite, enquanto que o "veículo" (leite sem proteína nem gordura), que em muitos países tem preços muito baixos, é remunerado aqui a preços mais altos (MADALENA, 2000a).

As médias \pm desvios padrão $(\mathrm{CV})$ da PROG e da PERG, no rebanho Mantiqueira foram, respectivamente, $76,59 \pm 31,92 \mathrm{~kg}(41,67 \%)$ e $3,43 \pm 0,64 \%$ (18,52\%). Estas estimativas foram menores que as obtidas por Bastos et al. (1999), na raça Pitangueiras, que foram 124,34 \pm 0,69 kg, para a produção de gordura, e 4,16 $\pm 0,31 \%$, para a porcentagem de gordura. Tais resultados, no entanto, devem ser observados com cautela, em razão do baixo número de observações.

As médias anuais para ambas as características são apresentadas na TABELA 4, revelando grandes oscilações durante o período analisado. Certamente, o nível de produção de leite e a disponibilidade de alimentos, tanto em qualidade quanto em quantidade, foram fatores

TABELA 3 - Médias de duração da lactação (DL) e erros-padrão (EP), de vacas do ecótipo Mantiqueira, segundo a estação de parto.

\begin{tabular}{ccc}
\hline Estação de Parto & Número de Observações & DL \pm EP(dias) \\
\hline Seca & 1636 & $242,85 \pm 2,75$ \\
Chuvosa & 1511 & $246,23 \pm 2,87$ \\
\hline
\end{tabular}


determinantes para tais oscilações.

Em relação às médias estacionais (TABELA 5), não foram verificadas quaisquer tendências de aumento ou diminuição nas características estudadas, em animais que pariram na época seca ou chuvosa.

\section{Período Seco}

O período seco pode ser definido como o tempo compreendido entre o final de uma lactação e o início da subseqüente, tendo grande influência no rendimento da produção de leite. $\mathrm{O}$ desempenho do animal em uma lactação está diretamente ligado à sua condição corporal ao parto, tornando-se necessária a existência de um período de descanso entre duas lactações subseqüentes para permitir a recuperação das reservas nutritivas (RIBAS et al., 1984) e a regeneração do tecido glandular secretor dos animais. Sorensen et al. (1993) afirmaram que a duração do período seco é um artifício com o qual o criador pode manipular a produção de leite e o seu rendimento financeiro. Secando-se uma vaca, há uma perda imediata de leite a qual o produtor espera recuperar na lactação seguinte. Essa relação entre o período seco e a produção de leite na lactação subseqüente é o parâmetro mais crítico para a avaliação econômica de diferentes períodos secos (DIAS \& ALLAIRE, 1982; SORENSEN et al., 1993).

A média \pm desvio padrão $(\mathrm{CV})$ do período seco precedente, no rebanho Mantiqueira, entre os anos de 1977 e 1997, foi de $256,40 \pm 180,29$ dias (70,32\%). A alta estimativa obtida para essa média pode ter sido reflexo da duração da lactação, isto é, curtas durações da lactação podem levar a maiores períodos secos, o que não é desejável, pois, de acordo com a literatura científica, períodos secos superiores a 60 dias não levam à produção de leite extra suficiente para compensar os custos de alimentação ou maximizar a produção por dia de vida útil do animal. Em relação ao período seco de acordo com o ano de parição, houve grande oscilação das médias ao longo dos anos, não apresentando tendência de aumento ou diminuição. As médias estacionais do período seco são apresentadas na TABELA 6 .

\section{Parâmetros genéticos}

As estimativas de herdabilidade e repetibilidade para as características PL, PROG, PERG, DL e PSECO, obtidas por meio de análises unicaracterísticas, bem como as correlações genéticas entre PL, PROG e PERG, estimadas por meio de análises bicaracterísticas, são apresentadas na TABELA 7.

TABELA 4 - Médias \pm erros-padrão da produção (PROG) e percentagem de gordura (PERG) de vacas do ecótipo Mantiqueira, segundo o ano de parto.

\begin{tabular}{ccccc}
\hline $\begin{array}{c}\text { Ano de } \\
\text { Parto }\end{array}$ & $\begin{array}{c}\text { Número de } \\
\text { Observações }\end{array}$ & $\begin{array}{c}\text { PROG } \pm \text { EP } \\
(\mathbf{k g})\end{array}$ & $\begin{array}{c}\text { Número de } \\
\text { Observações }\end{array}$ & $\begin{array}{c}\text { PERG } \pm \text { EP } \\
(\%)\end{array}$ \\
\hline 77 & 115 & $39,60 \pm 5,15$ & 104 & $3,43 \pm 0,10$ \\
78 & 99 & $55,62 \pm 5,55$ & 88 & $3,44 \pm 0,11$ \\
79 & 54 & $70,09 \pm 7,50$ & 53 & $3,28 \pm 0,15$ \\
80 & 116 & $52,19 \pm 5,15$ & 112 & $3,42 \pm 0,10$ \\
81 & 136 & $55,45 \pm 4,72$ & 133 & $3,48 \pm 0,09$ \\
82 & 84 & $50,07 \pm 6,01$ & 82 & $3,29 \pm 0,12$ \\
83 & 39 & $29,41 \pm 8,83$ & 36 & $2,73 \pm 0,18$ \\
\hline
\end{tabular}

TABELA 5 - Médias de produção (PROG) e percentagem de gordura (PERG) e respectivos erros-padrão (EP), de vacas do ecótipo Mantiqueira, segundo a estação de parto.

\begin{tabular}{ccccc}
\hline $\begin{array}{c}\text { Estação de } \\
\text { Parto }\end{array}$ & $\begin{array}{c}\text { Número de } \\
\text { Observações }\end{array}$ & PROG \pm EP $(\mathbf{k g})$ & $\begin{array}{c}\text { Número de } \\
\text { Observações }\end{array}$ & PERG \pm EP (\%) \\
\hline Seca & 326 & $45,60 \pm 2,18$ & 306 & $3,47 \pm 0,04$ \\
Chuvosa & 317 & $46,41 \pm 2,53$ & 301 & $3,45 \pm 0,05$ \\
\hline
\end{tabular}

Ciênc. agrotec., Lavras, v. 30, n. 3, p. 529-537, maio/jun., 2006 
TABELA 6 - Médias de período seco (PSECO) e respectivos erros-padrão (EP), de vacas do ecótipo Mantiqueira, segundo a estação de parto.

\begin{tabular}{ccc}
\hline Estação de Parto & Número de Observações & PSECO \pm EP (dias) \\
\hline Seca & 1558 & $252,57 \pm 5,07$ \\
Chuvosa & 1456 & $248,49 \pm 5,60$ \\
\hline
\end{tabular}

TABELA 7 - Herdabilidades (na diagonal), repetibilidades $(\hat{r})$ e correlações genéticas (acima da diagonal) entre produção total de leite (PL), produção de gordura (PROG), percentagem de gordura (PERG), duração da lactação (DL) e período seco (PSECO).

\begin{tabular}{ccccccc}
\hline Característica & PL & PROG & PERG & DL & PSECO & $\hat{r}$ \\
\hline PL & 0,25 & 0,36 & $-0,02$ & - & - & 0,38 \\
PROG & - & 0,09 & $-0,04$ & - & - & 0,09 \\
PERG & - & - & 0,00 & - & - & 0,00 \\
DL & - & - & - & 0,16 & - & 0,28 \\
PSECO & - & - & - & - & 0,06 & 0,16 \\
\hline
\end{tabular}

A estimativa de herdabilidade para a característica PL foi 0,25 , sendo próxima à obtida, na raça Caracu, por Pereira \& Pereira (1984). No entanto, é inferior as mencionadas por Quirino (1994), nas raças Pitangueiras e Caracu, respectivamente. A estimativa de herdabilidade encontrada para PL no presente estudo, indica que pode ocorrer razoável resposta à seleção, em virtude da variabilidade genética existente, ao contrário das características PROG, DL e PSECO, cujas estimativas de herdabilidade indicam que a seleção para essas características seria pouco efetiva. Para a PERG, em virtude da inexistência de variação genética aditiva na população $\left(h^{2}=0,0\right)$, não seriam obtidos ganhos genéticos, em programas de seleção.

O valor do coeficiente de repetibilidade $(\hat{r})$ estimado para PL $(0,38)$ sugere a possibilidade de seleção de vacas para PL nas primeiras lactações, ao contrário da PROG e PERG, em que a baixa repetibilidade indica a necessidade de se tomar mais medidas de ambas características para melhorar a precisão da seleção.

Na TABELA 7 são apresentadas as correlações genéticas entre PL e as características PROG e PERG. Os baixos valores encontrados indicam que a maioria dos genes que atuam sobre a PL não apresentam efeitos sobre PERG, sugerindo que a seleção para PL não resultaria em alterações na PERG. Entretanto, a correlação genética entre PL e PROG foi moderada e positiva, sugerindo associação favorável entre elas, podendo-se concluir que a seleção para produção de leite pode elevar a quantidade de gordura produzida na lactação. Todavia, o valor encontrado nesse estudo é inferior aos freqüentemente mencionados na literatura, principalmente quando comparado aos valores relatados para raças especializadas na produção de leite.

\section{CONCLUSÕES}

O valor de herdabilidade obtido para a produção de leite indica a existência de variabilidade genética aditiva na população.

Os resultados obtidos para as características produção de gordura e porcentagem de gordura indicam que baixos ganhos genéticos seriam obtidos pela seleção e que medidas de melhorias no manejo e na alimentação poderiam ser mais expressivas na melhoria dessas características.

A correlação genética entre a produção total de leite e produção de gordura, sugere que a seleção para produção de leite resultaria, indiretamente, em aumento na produção de gordura.

\section{REFERÊNCIAS BIBLIOGRÁGICAS}

ABREU, O. Programas de mejoramiento genético ejecutados por el Fondo Nacional de Investigaciones Agropecuárias en la Cuenca de Lago de Maracaibo. Revista Ciencia y Tecnologia Venezuela, Caracas, v. 5, n. 2, p. 27 29, 1988. 
ALBA, J.; KENNEDY, B. Genetic parameters of purebred and crossbred milking Criollos tropical Mexico. Animal Production, Edinburgh, v. 58, n. 3, p. 158-165, 1995.

BASTOS, J. F. P.; LOBO, R. B.; DUARTE, F. A. M.; SCHWENGBER, E. B. Efeito do pai do feto sobre as características produtivas e reprodutivas de vacas da raça Pitangueiras. Revista Brasileira de Zootecnia, Viçosa, v. 28, n. 5, p. 982-986, 1999.

BODISCO, V.; ABREU, O. Producción de lache por vacas Criollas puras. In: HODGES, J. (Ed.). Recursos genéticos animales en América Latina. Roma: FAO, 1981. p. 17-39.

BOLDMAN, K. G.; KRIESE, L. A.; VLECK, L. D. van; TASSELL, C. P. van; KACHMAN, S. D. A manual for use of MTDFREML: a set of programs to obtain estimates of variances and covariances [DRAFT]. Lincoln: USDA/ARS, 1995. $125 \mathrm{p}$.

CUNNINGHAM, E. P.; SYRSTAD, O. Crossbreeding Bos indicus and Bos taurus for milk production in the tropics. Food Agricultural Organic Animal Production, Rome, n. 68, 1987.

DIAS, F. M.; ALLAIRE, F. R. Dry period to maximize milk production over two consecutive lactations. Journal Dairy Science, Champaign, v. 65, n. 1, p. 136-145, 1982.

DICKERSON, G. E. Experimental approaches in utilizing breed resources. Animal Breeding, [S.1.], v. 37, p. 191, 1969. Abstract.

JORDÃO, L. P. Estudo retrospectivo e comparativo de dados sobre bovinos de raças Caracu e Mocha Nacional. Boletim de Indústria Animal, Nova Odessa, v. 15, p. 23-58, 1956.

MADALENA, F. E. Valores econômicos para a seleção de gordura e proteína do leite. Revista Brasileira de Zootecnia, Viçosa, v. 29, n. 3, p. 678-684, 2000a.

MADALENA, F. E. Conseqüências econômicas da seleção para gordura e proteína do leite. Revista Brasileira de Zootecnia, Viçosa, v. 29, n. 3, p. 685-691, 2000b.

MADALENA, F. E. Produção de leite e intervalo entre partos de vacas HPB: Gir num alto nível de manejo. Pesquisa
Agropecuária Brasileira, Brasília, v. 18, n. 2, p. 195-200, 1983.

MAGOFKE, S.; ALBA, J.; MUÑOZ, H. Informe del peogresso sobre mejoramiento genético de ganado Criollo Lechero en Maracay, Venezuela, entre los años 1955-64. Men. Asociate Latinoamericano Produto Animal, [S.l.], v. 1, p. 105-127, 1966.

MAGOFKE, S.; BODISCO, V. Estimaciones del mejoramiento genético de ganado Criollo Lechero de Turralba. Men. Asociate Latinoamericano Produto Animal, [S.1.], v. 1, p. 77-103, 1966.

MELLO, A. A. Análise de seleção ao nível de fazenda para características leiteiras e reprodutivas em um rebanho da raça Gir. 1994. 98 f. Dissertação (Mestrado) Universidade Federal de Minas Gerais, Belo Horizonte, 1994.

PEREIRA, J. C. C. Estudo da relação entre características produtivas e reprodutivas de um rebanho bovino nativo da raça Caracu. 1993. 128 f. Tese (Doutorado) - Universidade Federal de Minas Gerais, Belo Horizonte, 1993.

PEREIRA, J. C. C.; PEREIRA, C. S. Estudo genéticoquantitativo de produção de leite em bovinos nativos da raça Caracu: I. produção de leite por dia de intervalo entre parto. In: REUNIÃO ANUAL DA SOCIEDADE BRASILEIRA DE ZOOTECNIA, 21., 1984, Belo Horizonte, Minas Gerais. Anais... Belo Horizonte: SBZ, 1984. p. 37.

PONCE, P.; BELL, L. Compacición de al leche y estudio de la lactancia en el Siboney de Cuba y sus parentales. Memoria ALPA, [S.1.], n. 16, p. 160, 1981.

QUIRINO, C. R. Parâmetros genéticos e de ambiente relacionados com características produtivas e reprodutivas de um rebanho da raça caracu. 1994. $86 \mathrm{f}$. Dissertação (Mestrado) - Universidade Federal de Minas Gerais, Belo Horizonte, 1994.

QUIRINO, C. R.; PEREIRA, J. C. C.; BERGAMANN, J. A. G.; PEREIRA, C. S. Uso da metodologia de modelos mistos na estimação de fatores ambientais e parâmetros genéticos em com características produtivas da raça Caracu. Archives Latinoamericano Produto Animal, [S.1.], v. 6, n. 1, p. 59-70, 1998. 
RIBAS, N. P.; MILAGRES, J. C.; CASTRO, A. C. G.; TORRES, C. A. A. Influência do intervalo de partos e do período seco sobre a produção de leite, gordura e porcentagem de gordura em rebanhos holandeses da bacia de Castrolandia, Estado do Paraná. Revista da Sociedade Brasileira de Zootecnia, Viçosa, v. 13, n. 2, p. 225-234, 1984.

SAS INSTITUTE. SAS user's guide: basic and statistics. Cary, 1985.

SILVA, M. V. G. B.; BERGMANN, J. A. G.; MARTINEZ, M. L. Associação genética, fenotípica e de ambiente entre medidas de eficiência reprodutiva e produção de leite na raça holandesa. Revista Brasileira de Zootecnia, Viçosa, v. 27, n. 6, p. 1115-1122, 1998.

SILVA, M. V. G. B.; FERREIRA, W. J.; COBUCI, J. A. Estimativas de tendência genética para características produtivas de um rebanho do ecótipo Mantiqueira. Revista Brasileira de Zootecnia, Viçosa, v. 30, n. 5, p. 1466-1475, 2001a.

SILVA, M. V. G. B.; MARTINEZ, M. L.; BERGMANN, J. A. G. Desempenho reprodutivo e produtivo da raça Holandesa em alguns estados brasileiros. Revista Ceres, Viçosa, v. 48, n. 277 , p. 293-309, 2001 b.

SORENSEN, J. T.; ENEVOLDSEN, C.; KRISTENSEN, T. Effects of different dry period lengths on production and economy in dairy herd estimated by stochastic simulation. Livestock Production Science, Amsterdam, v. 33, p. 77-90, 1993.

TEODORO, R. L.; LEMOS, A. M. Cruzamentos de boninos para producción de lenche y carne. In: . Avances en la produccion de leche y carne en el tropico americano. Santiago: FAO, 1992. cap. 6, 503 p. 